Rabaska

Revue d'ethnologie de l'Amérique française

BOUCHARD, MARTIN. Léon Bouchard. Sculpteur du

Lac-Saint-Jean. [Préface de JEAN-FRANÇOIS BLANCHETTE].

Salaberry-de-Valleyfield, Marcel Broquet, éditeur,

" Signatures », 2015, 103 p. ISBN 978-2-89726-017-0

\title{
Bernard Genest
}

Volume 14, 2016

URI : https://id.erudit.org/iderudit/1037468ar

DOI : https://doi.org/10.7202/1037468ar

Aller au sommaire du numéro

Éditeur(s)

Société québécoise d'ethnologie

ISSN

1703-7433 (imprimé)

1916-7350 (numérique)

Découvrir la revue

Citer ce compte rendu

Genest, B. (2016). Compte rendu de [BouCHARD, MARTIN. Léon Bouchard.

Sculpteur du Lac-Saint-Jean. [Préface de JEAN-FRANÇOIS BLANCHETTE].

Salaberry-de-Valleyfield, Marcel Broquet, éditeur, «Signatures », 2015, 103 p.

ISBN 978-2-89726-017-0]. Rabaska, 14, 230-233.

https://doi.org/10.7202/1037468ar d'utilisation que vous pouvez consulter en ligne. 
Bastien et Levert publient Québec à l'été 1950 en 1982, une sélection de 166 photos, tirées du corpus de plus de 3000 photos de Moser, présentées par Roch Carrier. Ce livre impressionnant par ses images pleine page, parfois à fond perdu, fait partie des beaux-livres publiés par cette maison d'édition alors qu'elle est toujours sous le contrôle de ces éditeurs. Le texte raconte la vie de Roch Carrier dans le Québec traditionnel et se sert des photographies comme illustration de ce que fut le Québec au milieu du Xx $x^{\mathrm{e}}$ siècle, encore fortement traditionnel, mais inspiré par les artistes et autres créateurs de changements. Carrier écrit, en épigraphe : «Le Québec au temps du muet, au temps du noir et blanc, au temps où la chrysalide allait devenir papillon, il fallait peut-être une étrangère pour le voir et pour nous le montrer avec intensité. » L'éditeur André Bastien m'a raconté que « les libraires d'alors ont boudé le livre, car les photos étaient en noir et blanc... » Il est désormais introuvable!

Quant à ceux qui mettront la main sur le beau catalogue publié par le MnBaQ, ils auront chez eux un livre durable qui inspirera certainement d'autres amateurs d'art, de photographies et de beaux livres, et voudront explorer à nouveau ce riche corpus de clichés pris par Lida Moser à l'été 1950.

Jean-François Blanchette Chercheur associé au Musée canadien de l'histoire

Bouchard, Martin. Léon Bouchard. Sculpteur du Lac-Saint-Jean. [Préface de Jean-François Blanchette]. Salaberry-de-Valleyfield, Marcel Broquet, éditeur, « Signatures », 2015, 103 p. ISBN 978-2-89726-017-0.

Ce livre est une « biographie artistique », nous dit l'auteur. On pourrait ajouter qu'il s'agit d'un récit de vie dont Martin Bouchard est l'interprète, l'histoire véritable n'étant ni narrée, ni écrite, mais révélée dans des ouvrages de bois et de pierre regroupés dans une espèce de musée à ciel ouvert, en pleine forêt. L'auteur, en effet, présente le parcours d'un homme ordinaire, son père, ancien forestier, qui a choisi de se raconter, non pas avec des mots, mais en façonnant des « mémoires dans le bois et le roc » pour reprendre une formule empruntée à Jean-François Blanchette dans la préface de l'ouvrage.

À la parole, Léon Bouchard a préféré la gouge et le ciseau. « Un jour, disait-il, les pierres vont parler pis personne pourra lés arrêter d'parler!» Son fils s'est fait un devoir de faire en sorte que le message soit entendu. Il l'a d'abord fait « connaître des gens du milieu des arts et des médias » qui l'ont soutenu dans son projet d'exposer les œuvres de son père dans des centres d'art 
et des musées. Ensuite il a pensé écrire un livre qui ferait connaître l'artiste auprès d'un large public. Martin Bouchard n'a jamais douté de l'importance de Léon Bouchard comme artiste populaire et comme artiste « tout court ». Pendant une dizaine d'années, il s'est appliqué à documenter les 275 sculptures que le sculpteur a réalisées au cours de sa vie: " Depuis les débuts, je documente son travail artistique en photographiant chacune des pièces. Vers 2005, j'ai commencé un tableau descriptif de toutes ses sculptures : titre[s], dimensions, essence[s] et date[s] de création, leur[s] propriétaire[s] et autres notes. \ C'est en travaillant le bois et la pierre que l'artiste se révèle mais, raconte son fils, il ne dédaignait pas commenter son travail devant les journalistes : «Mon père était intarissable dans ses entrevues, et faisait le bonheur des journalistes [...] Chanceux, j'avais en main la plupart de ces entrevues in extenso. J'ai donc choisi de lui laisser souvent la parole pour expliquer ses œuvres. » Témoin privilégié du parcours de l'artiste, Martin Bouchard a rassemblé tout le matériel documentaire pouvant éclairer l'œuvre et la replacer dans ses contextes : «J'avais tout en main à l'ordinateur et dans mes tiroirs. Je n'avais qu'à tout mettre ça dans un plan et rédiger » explique-t-il.

La passion pour la sculpture arrive relativement tard dans la vie de Léon Bouchard. Né en 1920, décédé en 2012, sa carrière de sculpteur s'étire de 1977 à 2009, mais l'essentiel de sa production se situe entre 1995 et 2004. C'est au moment de prendre sa retraite alors que, pour la première fois de sa vie, il peut envisager occuper son temps comme il l'entend, qu'il commence à s'intéresser à la sculpture. Cet intérêt se développe en même temps que prend forme un autre projet, celui de se construire un chalet en forêt : il le baptisera Petit bonheur, un nom qui évoque la quête d'un rêve, sans doute davantage en devenir que véritablement atteint. Situé à quelques kilomètres de la maison, ce chalet de bois rond devient le lieu de rassemblement de la famille, mais aussi une retraite où l'artiste trouve dans la nature environnante la source de son inspiration. Le projet de musée, bien qu'informel, prend forme petit à petit, alors que l'ancien forestier aménage son terrain et y trace des sentiers qui sont prétextes à la découverte d'autant de « Monstres, Baleine à bosses, Pieuvre, Requin mangeur d'hommes [...]». Ailleurs ce sont les «bêtes d'eau » où « Il installe alors le long du ruisseau Phoque, Loutre, Ours polaire et Harfang des neiges. » Ailleurs encore, c'est un oratoire dédié au Sacré-Cœur et à Sainte-Hedwidge, la patronne du village où il est né, qu'il élève à l'orée du bois. Pendant les mois d'hiver, c'est dans l'atelier installé au sous-sol de la maison, qu'il sculpte des ouvrages qu'il expose ensuite à son chalet, dans une « vitrine » spécialement aménagée à cet effet, de même que dans une construction de pierre des champs qu'il appelle son «musée ». L'ensemble forme un tout cohérent, une espèce de parc ou de musée à ciel 
ouvert. Le projet n'est pas sans rappeler celui d'un Roger Ouellet (19161999) qui, à Sainte-Agnès-de-Charlevoix, avait érigé sur sa terre monuments et sculptures fabriqués de matériaux aussi divers que le bois, le ciment, le métal et la pierre. On pourrait aussi faire le lien avec la «forêt enchantée » d'Aimé Desmeules (1917-1997) de Saint-Paul-de-la-Croix, qui révélait aux promeneurs un monde fantastique de bêtes surgissant à chaque détour d'un sentier aménagé en pleine forêt. Mais la persévérance du sculpteur, qui n'avait de cesse d'extraire du sol de grosses roches qu'il transformait en œuvres d'art, me fait également penser au facteur Cheval (Ferdinand Cheval, 1836-1924) de Hauterives, en Drôme (France). Architecte du naïf, Cheval consacra tous ses temps libres à édifier son Palais idéal et son Tombeau du silence et du repos sans fin, deux chefs d'œuvre d'architecture classés au titre de monuments historiques en dépit des protestations des fonctionnaires du ministère de la Culture. L'œuvre de Bouchard n'est certes pas aussi monumentale que celle de Cheval, mais l'un et l'autre participent du même acharnement dans la poursuite de leur rêve. Ils se rejoignent aussi dans ce que qu'ils tirent de la nature le matériau nécessaire à la construction de leur œuvre.

Martin Bouchard est de toute évidence un grand admirateur de Léon Bouchard, homme de trente-six métiers, bûcheron, draveur, charretier, soldat malgré lui, menuisier, charpentier, aviculteur, journalier, entrepreneur et, pour couronner le tout, sculpteur. D'ailleurs, l'auteur est clair à ce propos : « Par ce livre, dit-il, je veux montrer toute l'admiration que j'ai toujours eu pour l'œuvre de mon père [...]. » Il semble évident que cette admiration dépasse largement la seule production artistique de Léon Bouchard. Celle-ci est manifeste dans chacun des chapitres du livre. On pourrait reprocher à l'auteur de n'avoir pas su prendre ses distances par rapport à son objet d'étude. Le dernier chapitre, paradoxalement intitulé « Analyse de son œuvre », fait davantage l'apologie de l'artiste plutôt que d'introduire une réflexion critique sur sa démarche. C'est ainsi qu'on peut lire que « Son art n'est pas parfait, mais il sait que sa Femelle d'orignal mettant bas est grandiose, que son Sacré-Cœur est majestueux, que sa Mère Thérésa est une formidable boule de piété, que La douceur fait rêver et qu'on a tous envie de s'abriter près d'Anne. Par contre, il ne sait pas qu'il touche au sublime avec sa Brebis, son Oiseau de proie, son Phoque, sa Crèche, son Maçon, son Patrimoine, son Lac Delaise, pour ne nommer que ceux-là. Car ici, nous ne sommes plus du tout dans l'art naif, mais dans l'art tout court. » Ce jugement n'est pas sans fondement, bien qu'un peu excessif. De nombreux spécialistes, ethnologues, muséologues, antiquaires, ont confirmé l'intérêt et la valeur de l'œuvre. On aurait préféré cependant que l'auteur se garde une petite gêne et qu'il permette au lecteur de se faire sa propre opinion. Il demeure que cette publication vient enrichir le 
répertoire des artistes populaires du Québec et contribuer à la connaissance, et à la reconnaissance, de l'un de ses représentants les plus originaux.

Bernard Genest

Société québécoise d'ethnologie, Québec

Bouchard, Russel. Chroniques d'histoire du Saguenay-Lac-Saint-Jean. Chicoutimi, Saguenay-Lac-Saint-Jean, 2011-2015, 3 tomes : t. 1, Du mythe à la réalité, 263 p. ISBN 978-2-921101-40-0; t. 2, Du rêve à la réalité, 2012, 410 p. ISBN 978-2-921101-41-7 ; t. 3, Devoir de mémoire, 2015, 445 p. ISBN 978-2-921101-43-1.

À l'heure des bilans, Russel Bouchard (prolifique auteur originaire de Chicoutimi-Nord, qui poursuit sa carrière sous le nom de Russel-Aurore Bouchard) nous livre une rétrospective de plus de quarante ans de carrière en recherche historique sur le Saguenay-Lac-Saint-Jean. Réuni en trois volumes parus entre 2011 et 2015, l'ensemble est composé d'articles inédits et d'autres pour la plupart initialement parus dans les pages de la revue d'histoire Saguenayensia. L'historienne s'engage dans une relecture personnelle du peuplement et du développement du pays intérieur. Ratissant très large, l'essayiste aborde autant les pages les plus sombres de l'histoire de la région que celles moins tragiques, mais tout aussi intéressantes, en plus de faire le portrait de certains personnages marquants. Elle a pris soin de rafraîchir les textes et de valider à nouveau les sources invoquées, " les dépoussiérer d'un rapide coup de plumeau littéraire» (t. 3, p. 9) pour fournir un contenu le plus actuel possible face aux connaissances et découvertes récentes.

Le premier tome, Du mythe à la réalité, rassemble treize essais et nous ramène au temps de la fondation de ce territoire. Le premier texte offre un survol historique de la région afin de permettre aux lecteurs d'avoir les bases factuelles pour bien suivre et comprendre les thèses développées dans les chroniques suivantes, lesquelles abordent notamment le processus de démocratisation de l'administration publique dans la vallée du Saint-Laurent ainsi que l'effondrement de la démocratie en Amérique septentrionale. L'auteure questionne ensuite le regroupement des Montagnais des contacts et la perception qui émane de la frontière entre sauvagerie et civilisation. Elle y reprend d'ailleurs la thèse controversée de la chasse-gardée de Tadoussac. Autre moment notable, elle rappelle un chapitre oublié de la période de la Nouvelle-France, la réduction de Métabetchouan, où l'on met à profit le concept assimilationniste élaboré par le père Le Jeune dans un programme de sédentarisation des Montagnais. L'auteure fait place dans son ouvrage à certaines catégories d'acteurs particulièrement importants, notamment aux 